\title{
PCR Detection of Mixed and Zoonoses Malaria Using Plasmodium spp Dynein Light Chain (dlc-tctex) Gene
}

\author{
Maureen W. Kariuki ${ }^{1,2}$, Elijah K. Githui ${ }^{2^{*}}$, Andrew G. McArthur ${ }^{3}$, \\ Rashid A. Aman ${ }^{4}$, Nyamu M. Njagi ${ }^{2}$, Agatha C. Mwangemi ${ }^{2}$ and Lucy W. Kamau ${ }^{2}$ \\ ${ }^{1}$ Pan African University of Basic Sciences/Jomo Kenyatta University of Agriculture and Technology, \\ P.O.Box 62000-00200, Nairobi, Kenya. \\ ${ }^{2}$ Molecular Genetics Laboratory, Institute of Primate Research, P.O.Box 24881, Karen, Kenya. \\ ${ }^{3}$ Department of Biochemistry and Biomedical Sciences, McMaster University, Hamilton, Ontario, \\ Canada. \\ ${ }^{4}$ African Center for Clinical Trials, P.O.Box 2288-00202, Nairobi, Kenya.
}

Authors' contributions

This work was carried out in collaboration among all authors. Author MWK was involved in samples collection, performed laboratory analysis and was involved in manuscript preparation. Author EKG was involved in concept development, design of the study, laboratory analysis and manuscript preparation. Author AGMA was involved in concept development, design of the study, laboratory protocol development and in manuscript preparation. Authors RAA and AGMA was involved in concept development, design of the study, laboratory protocol development and in manuscript preparation. Author NMN was involved in samples collection and performed laboratory analysis. Author ACM was involved in samples collection and performed partial laboratory analysis. Author LWK was involved in samples collection. All authors read and approved the final manuscript.

Article Information

DOI: $10.9734 / A R R B / 2019 /$ v32i430091 Editor(s):

(1) Dr. J. David Puett, Professor, Department of Biochemistry and Molecular Biology, University of Georgia, Athens, USA.

Reviewers:

(1) hmed Tabbabi, Jichi Medical University, Japan.

(2) K. Ramesh Kumar, K. N. R. University of Health sciences, India.

(3) Franco Cervellati, University of Ferrara, Italy. Complete Peer review History: http://www.sdiarticle3.com/review-history/50344 


\begin{abstract}
Novel gene targets are needed in accurate diagnosis of malaria. Previous studies show that the dynein light chains $(d / c)$ in Plasmodium are uniquely conserved within the species, possibly due to their role as the cargo adptor moiety. This study aimed at the development of PCR assay for the detection of Plasmodium based on the (dlc-Tctex) as a genus and species-specific tool in malaria diagnosis. Multiple primers were designed based on Plasmodium spp dlc(Tctex) genes. The primers were applied on PCR to detect malaria on clinical samples and on laboratory maintained isolates of $P$. falciparum and $P$. vivax for human infecting species and $P$. knowlesi and $P$. cynomolgi for zoonoses infection involving primates. The amplified PCR fragments were gene cleaned and sequenced. BLASTn e-values output from the raw nucleotide queries supports that the genes are uniquely conserved. Species-specific primers amplified $P$. falciparum infections with no crossreactivity to $P$. vivax, $P$. knowlesi or $P$. cynomolgi species. In this assay only 11 out of the 30 microscope positive malaria positive clinical blood samples were positive for PCR detection of $P$. falciparum infection. Primers designed for Plasmodium genus amplified the target band in all clinical malaria samples but also had another specific band amplification. This preliminary data demonstrate that a species-specific dlc(Tctex) PCR assay can be used for detection of $P$. falciparum and optimized genus primers can be applied to differentiate mixed malaria infections.
\end{abstract}

Keywords: Plasmodium malaria; PCR detection; dlc-Tctex.

\section{INTRODUCTION}

Malaria infection is a major public health problem in tropical and subtropical regions of the world. Pregnant women and children below five years of age are at a higher risk of malaria than adults due to their relatively low immunity [1-5]. The disease is caused by infection of red blood cells by protozoan parasites of the genus Plasmodium that is inoculated into the human host by female anopheline mosquito while feeding [5,6]. The four Plasmodium species that infect humans are $P$. falciparum, $P$. vivax, $P$. malariae, and $P$. ovale. $P$. falciparum and $P$. vivax are the most widespread and common causes of mixed-species malaria [7]. Increasingly, human infections with the non-human primate malaria parasites $P$. cynomolgi and $P$. knowlesi are being reported in South East Asia [8-12]. P. knowlesi has a 24-hour asexual life cycle, the shortest observed thus far, for humaninfecting parasites. This short cycle can lead to rapid increases in parasitaemia hence severe disease including fatalities as reported in recent studies [11]. Microscopically, P. knowlesi exhibits stage-dependent morphological similarities to $P$. malariae and these similarities have contributed to misdiagnosis of $P$. knowlesi as $P$. malariae, a relatively benign disease $[11,13]$.

The first symptoms of malaria are non-specific and similar to the symptoms of a minor systemic viral infection: headache, lassitude, fatigue, abdominal discomfort, muscle and joint aches, usually followed by fever, chills, perspiration, anorexia, vomiting and worsening malaise [1317]. Malaria is thus frequently over-diagnosed on the basis of symptoms alone, especially in endemic areas. Conversely, in missed diagnosis malaria cases, delayed treatment or wrong prescription of medicines for for $P$. falciparum cases, the parasite burden rises in the lessimmune individuals and severe malaria may occur within a few hours. At this stage, fatality in people receiving treatment is typically $10 \%$ to $20 \%$ and if further delayed, severe malaria is fatal in the majority of cases $[18,19]$.

Recommended methods for routine diagnosis of malaria are the observation of parasite in peripheral blood parasite by microscopy and detection of parasite antigens by rapid diagnostic tests (RDTs) [20]. There has been observed limitations in these methods in differentiating mixed-species malaria infections. This has impact on therapeutic decisions, including selection, dose, and timing of antimalarial drugs. Treatment for a single species, rather than multiple species, can have serious clinical consequences and the current reports of zoonoses infections is a concern $[8,11]$. In addition, mixed-species infections are related to the growing problem of anti- malarial drug resistance, which can enable emergence of drug-resistant clones of Plasmodium parasites and relapse infection [20-22]. In recent years, several polymerase chain reaction assays have been developed and evaluated for malaria diagnosing, most often based on species- 
specific sequences of the parasites' $18 \mathrm{~S}$ small subunit rRNA gene [23-25]. In our previous in silico study done on the Plasmodium spp. dynein light chain genes, the dlc-TcTex1 genes were found to be conserved within the species and had low similarity to human orthologues [26]. These differences can be attributed to variations in the cargo attached via the dlc adaptors to the main dynein motor complex [27]. In this study we analyze application of dlc-Tctex gene in PCR detection of Plasmodium spp as an additional tool for differentiating mixed malaria and zoonoses infections.

\section{MATERIALS AND METHODS}

\subsection{Laboratory Derived Samples}

Extracted DNA sample of laboratory culture isolates or laboratory animal propagated plasmodial parasites were kindly provided: $P$. falciparum (David Peterson, University of Georgia, USA), P. Knowlesi (Julian Rayner, Sanger Institute, UK), P. vivax (Robert Moon, London School of Hygiene and Tropical Medicine (LSHTM), UK), and $P$. cynomolgi (Jenneby Mamun, Institute of Primate Research, Kenya (IPR)).

\subsection{Clinical Samples}

Ethical clearance for sampling blood from human subjects was obtained from Kenyatta National Hospital/University of Nairobi Ethics Committee $(\mathrm{KNH} / \mathrm{UON})$. Sampling was done in coast region of Kenya endemic to malaria. This study involved patients who went freely for clinical check up at Coast General hospital and Sacred Medical clinic, Mombasa. In presumptive malaria cases, approximately 500 ul (microliter) of blood was collected in EDTA vaccutainer (Benton and Dickson), an aliquot for microscopy observation separated, and the rest of the blood was stored in the laboratory at $-20^{\circ} \mathrm{C}$. Laboratory tests by microscopy were carried out where 42 samples were classified as malaria positive but only 30 of these samples were nonambiguous.

\subsection{Ethics Statement}

This research project was conducted with the approval of both Institute of Primate Research/National Museums of Kenya scientific committee and Pan African University of Basic Sciences-Jomo Kenyatta University of Agriculture and Technology graduate students project approval committee. Due to the use of human subjects, clearance was sought from $\mathrm{KNH} / \mathrm{UON}$ ethics committee (above) in accordance to the Helsinki Declaration of 1975, as revised in 2000. Patients coming to the clinic were informed about the study through posters and personal contact and the study objectives were explained in English or in Kiswahili (vernacular). Patients expressing interest to participate got more information on potential risks. Questionnaire form cleared by $\mathrm{KNH} / \mathrm{UON}$ ethics committee was applied to check subjects' understanding that the study intent is to improve on malaria diagnosis. For individuals under 18 years, parents gave the consent to participate by signing on behalf.

\subsection{DNA Extraction}

$200 \mathrm{ul}$ of blood was aliquoted into $1.5 \mathrm{ml}$ microcentrifuge tubes for DNA extraction. Briefly, 20 ul ProteinaseK was added to sample and $200 \mathrm{ul}$ of digestion/lysis buffer and incubated at $56^{\circ} \mathrm{C}$ water-bath. DNA was bound to silica matrix spin column, washed and eluted in 100 ul-to-200 ul nuclease-freee distilled de-ionized water, as described in owner's manual (Invitrogen, Grand Island, NY 14072, USA ) and stored at $-20^{\circ} \mathrm{C}$.

\subsection{Primers and PCR}

$P$. falciparum specific primers were designed based on Plasmodium dlc gene sequences obtained from NCBI nucleotide database $[27,28]$. These were aligned by Clustal-W software in the BioEdit Suite (BioEdit v7.0.5 copyright (c) 19972005) against human and related dlc sequences (Supplementary data, Table 1). Similarly, Plasmodium genus and species specific primer sets were designed based on respective conserved sequences. PCR amplification was done using $100 \mathrm{ng}$ of the template DNA, 0.5uM of each primer, $200 \mathrm{uM}$ each dNTP, 10X buffer with $2.0 \mathrm{mM} \mathrm{MgCl} 2$ and $0.5 \mathrm{U}$ Takara Taq polymerase (Takara Inc. Japan), made up to 25ul with ddH2O. Cycling conditions: Taq polymerase activation $92^{\circ} \mathrm{C}, 3 \mathrm{~min} ; 35$ cycles profile:- denaturation, $92^{\circ} \mathrm{C}, 1 \mathrm{~min}$; annealing, $52^{\circ} \mathrm{C}$-to- $57^{\circ} \mathrm{C}, 1 \mathrm{~min}$; Taq polymerase nucleotide elongation, $70^{\circ} \mathrm{C}, 1 \mathrm{~min}$; final polymerase extension step, $70^{\circ} \mathrm{C}, 3 \mathrm{~min}$ and final storage in the PCR machine at $8^{\circ} \mathrm{C}$.

\subsection{Gene Clean and Sequencing}

The different Plasmodium species control samples and clinical blood samples that were positive for malaria by PCR using $P$. falciparum 
specific and genus specific primers were reamplified for purposes of sequencing. PCR products were analyzed on $1 \%$ agarose gel and the DNA viewed via ethidium-bromide fluorescence in utra-violet transluminator. The target fragments were excised from the gel and dissolved in sodium iodide solution at $56^{\circ} \mathrm{C}$, then applied to the purification column (innuPREP gel extraction kit, Lifescience, Jena, Germany). DNA was eluted in 35 ul nucleasefree ddH2O. Eluted fragments were sent (Macrogen Inc. Netherlands) for custom sequencing in 454 Sanger's di-deoxy sequencing procedures.

\subsection{Raw Sequence Editing and BLASTn Procedures}

Raw sequences were Clustal-W aligned in BioEdit suite program and 20-to-30 nucleotides at the 5'end and 3'end trimmed. Consensus sequence derived from three repeat sequences was applied in BLASTn analysis. Representative sequences were used as queries against NCBI non-redundant nucleotide databases using BLASTn $[28,29]$ at default algorithm parameters (expectation thresh hold of $10)$ to identify orthologous and paralogous genes.

\section{RESULTS}

\subsection{P. falciparum Specific PCR Products}

$P$. falciparum specific primers $\mathrm{PCR}$ performed on different Plasmodium species control and clinical samples produced a band approximately 300bps. 3D7 P. falciparum laboratory culture controls and some of clinical samples positive for malaria had distinctive PCR product while others had faint or no bands. (Fig.1a). Out of the 30 clinical blood samples which had been found to be positive for malaria through microscopy, only 11 had clear positive for $P$. falciparum by PCR. The samples re-amplified for sequencing purposes are shown (Fig. 1b).

\subsection{Genus Specific PCR Products}

PCR performed using genus-specific primers produced variant size band in the respective species. P. falciparum (3D7 and Dd2), P. vivax, $P$. cynomolgi and $P$. knowlesi control samples produced a band approximate of $300 \mathrm{bps}$ (Fig. 2 (A), (B), (C)) but $P$. vivax, $P$. cynomolgi and $P$. knowlesi produced a band approximately 400bps. Experimental primates derived $P$. knowlesi and $P$. vivax and some P.falciparum samples also produced approximately 700bps and 1000bps product. The extra PCR products in $P$. knowlesi and $P$. falciparum samples could be eliminated by the application of stringent PCR conditions but remained persistent in all $P$. vivax positive samples (Fig. 2 (c)).

When these primers were applied in clinical human blood samples, they produced at least one band in the clinical samples that corresponded to the control samples (270bps to- $300 \mathrm{bps}$ ) product but also amplified another gene target approximately 1000bps band (Fig. 3(A), (B), (C)). These target PCR products (Fig. 4 $(A)$, (B)) were gene cleaned for sequencing purposes (Fig. $4(\mathrm{C})$ ).

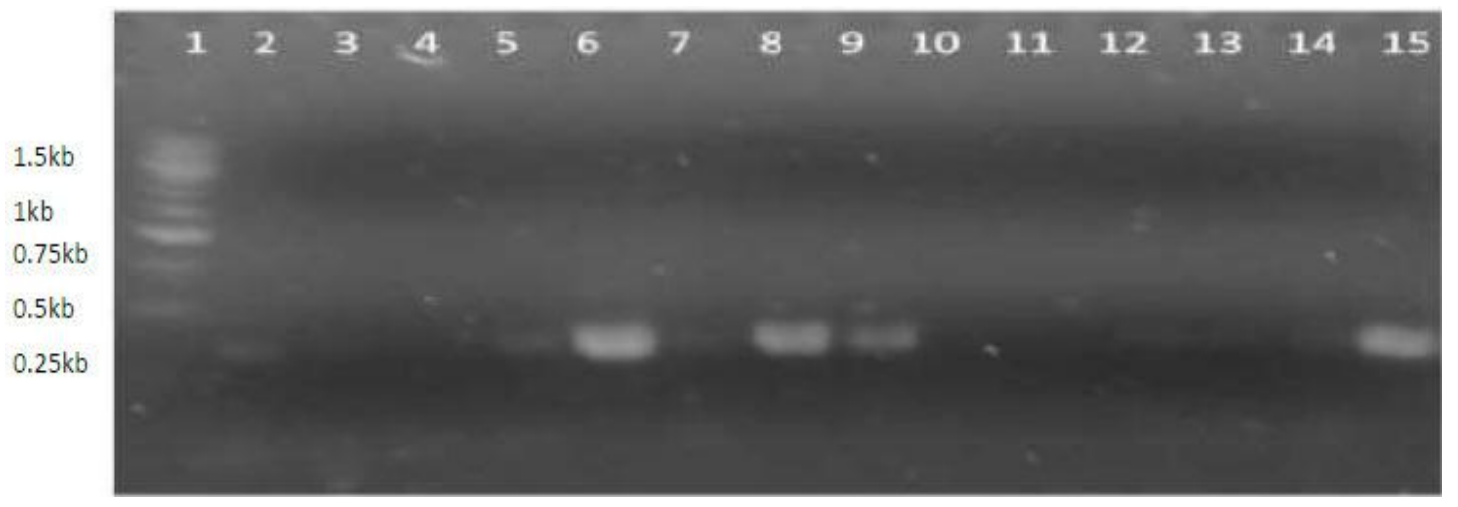

Fig. 1a. PCR products (P. falciparum specific primers). Lane 1; Mol wt marker; clinical malaria samples: lane 2; KS3, lane 3; KS4, lane 4; H9C, lane 5; K10, lane 6; Dd2; lane 7, PR26D; lane 8, 3D7; lane 9, Mumias1; lane 10, Mumias2; lane 11, G9; lane 12, B3; lane 13, B1; lane 14, PR26D; lane15, H9C 


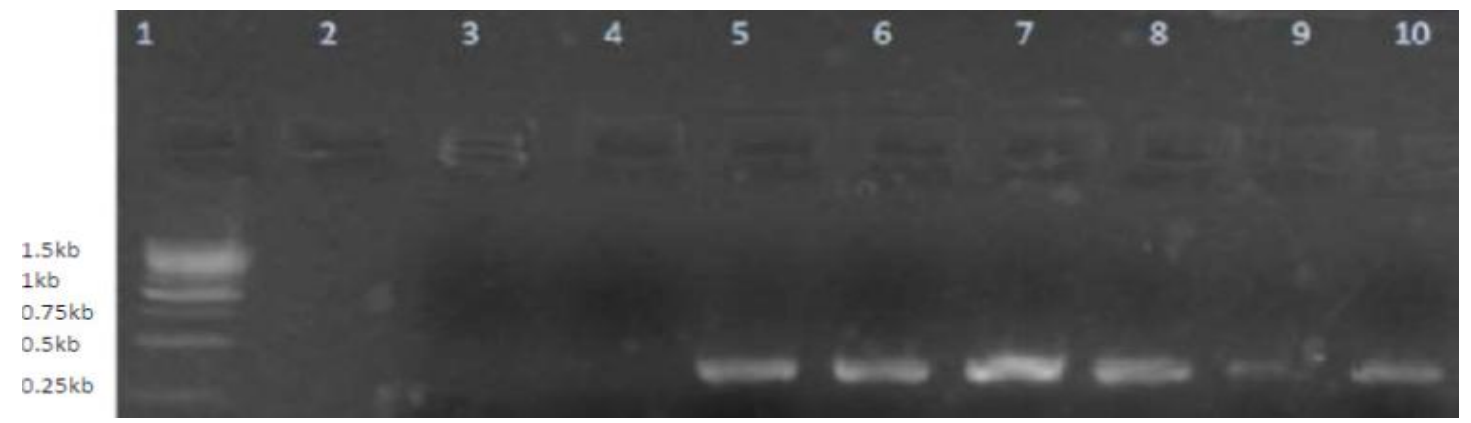

Fig. 1b. (Gene Clean products): Lane 1, Mol wt marker; lane 2, buffer only; lane 3 \& 4 Malaria ve human DNA samples, lane 5, 3D7 control; lane 6-10 clinical malaria samples
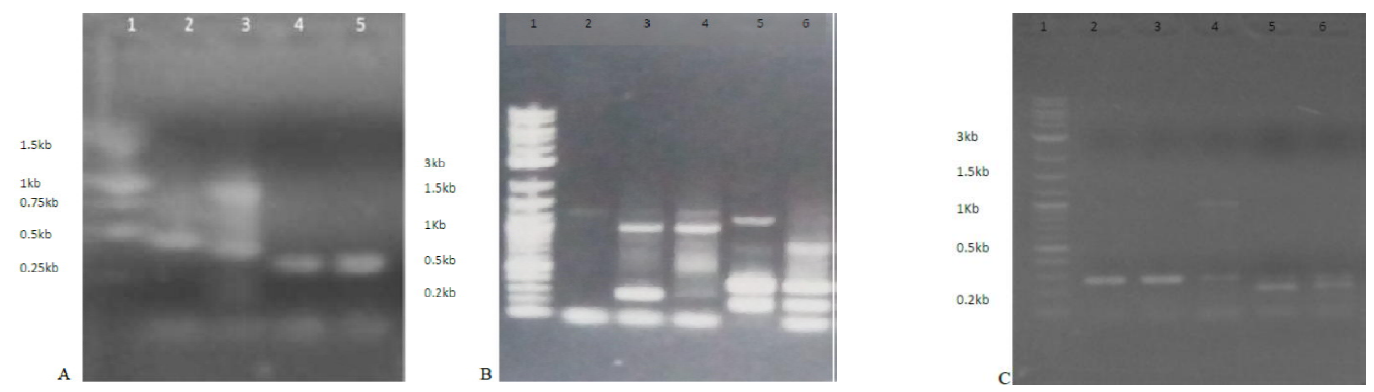

Fig. 2. (A). PCR (Genus primers). Lane 1; Mol wt marker, lane 2; $P$. cynomolgi, lane 3; $P$. vivax, lane 4; P. falciparum (3D7), lane 5; P. falciparum (Dd2); (B). PCR (Genus primers). Lane 1; Mol wt marker, lane 2, Malaria (negative), lane 3; $P$. falciparum (Dd2); lane 4, $P$. vivax; lane 5; $P$.

kowlesi (Sanger Institute), lane 6; P. knowlesi (IPR). (C). PCR (Genus primers, strigent conditions PCR ). Lane 1, Mol wt marker; lane 2, P. falciparum (3D7); lane 3; P. falciparum (Dd2); lane 4, P. vivax; lane 5; P. knowlesi (Sanger Institute), lane 6; P. knowlesi (IPR)
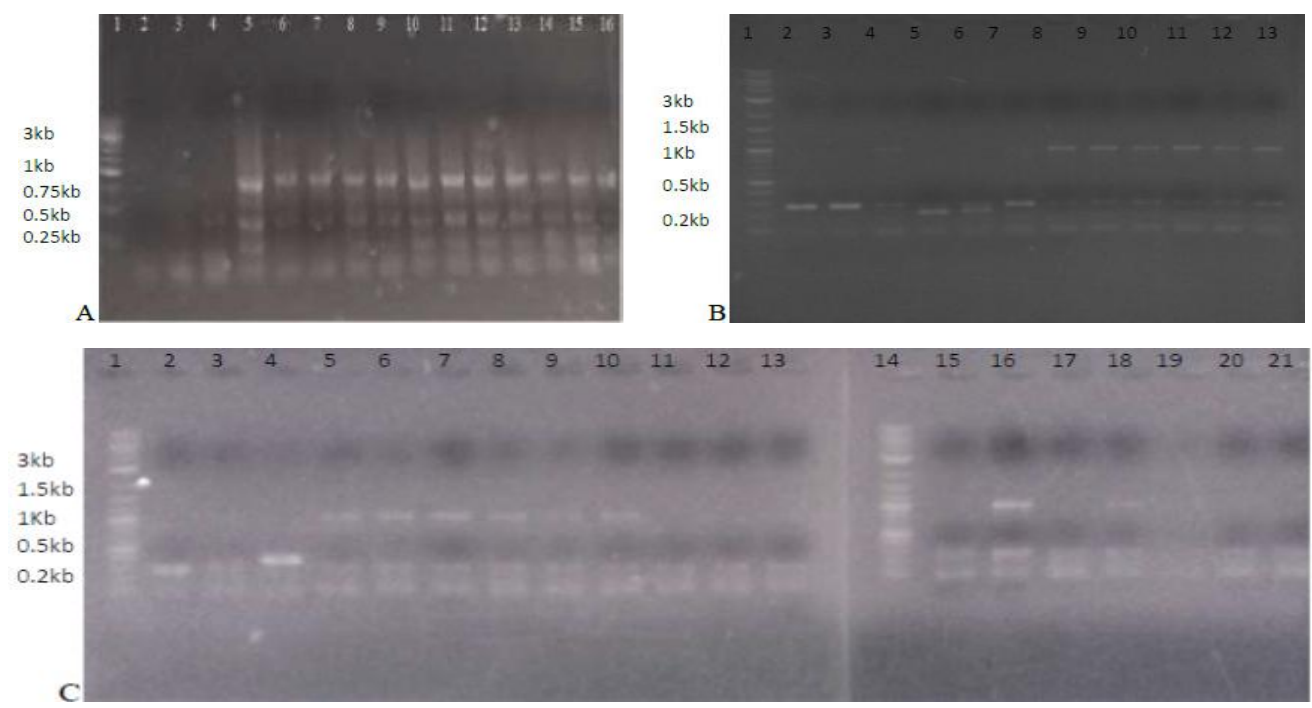

Fig. 3 (A): Clinical samples PCR (Genus primers). Lane1, Mol wt maker; Lane2, Malaria -ve sample; Lane3, P. vivax; Lane4, P. cynomolgi; Lane 5- to- 16 (clinical samples) (B): Clinical samples PCR (Genus primers). Lane 1, Mol wt marker; lane 2, P. falciparum (3D7); lane 3; $P$. falciparum (Dd2); lane 4, P. vivax; lane 5; $P$. knowlesi (Sanger Institute), lane 6; $P$. knowlesi (IPR); lane 7- to- 13 (clinical samples) (C): Varied clinical samples PCR (Genus primers). Lane 1 and 14, Mol wt marker; lane 2, P. falciparum (3D7); lane 3, P. vivax; lane 4; $P$. knowlesi (Sanger Institute); lane 5- to- 13 and 15 to- 21 (clinical samples) 

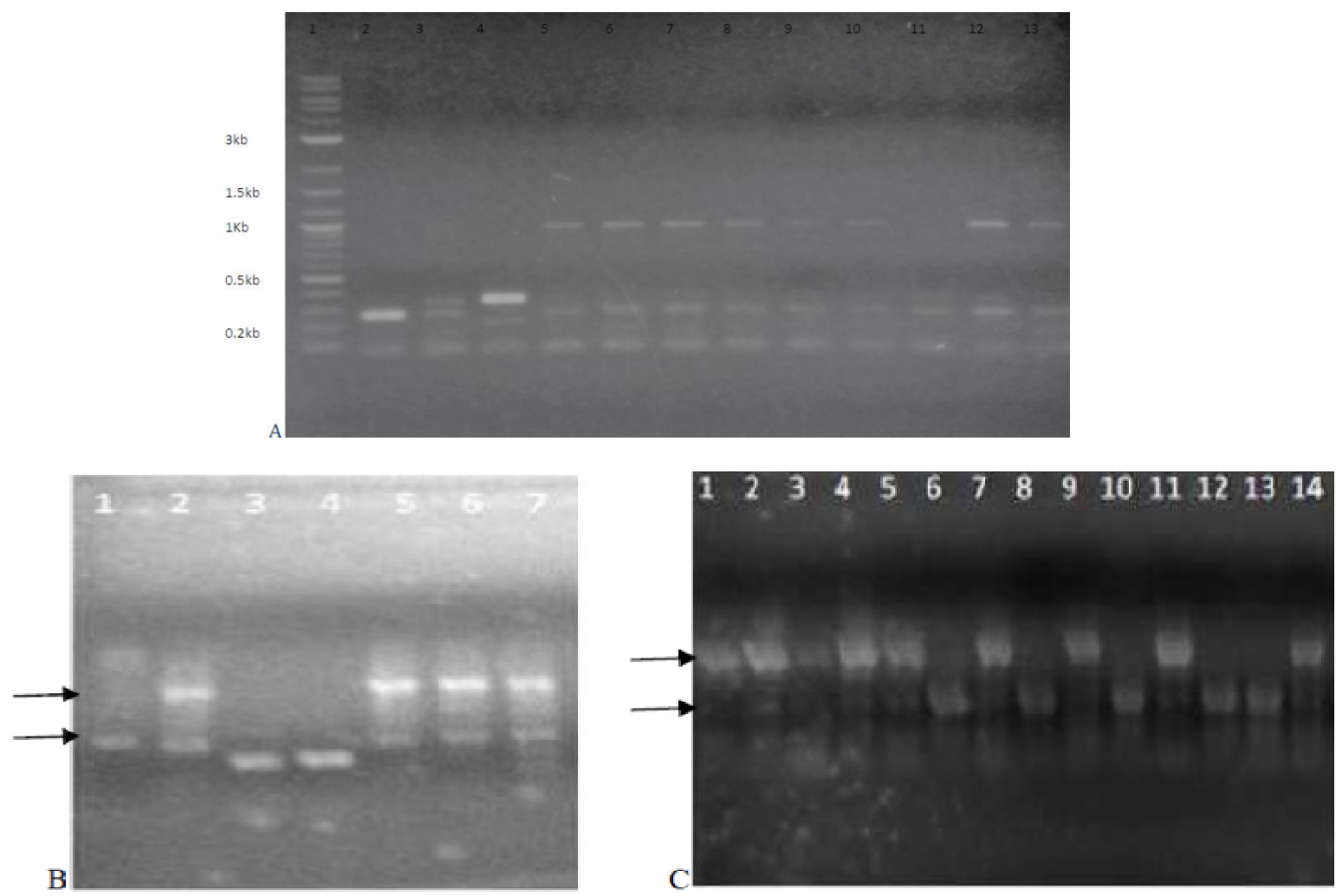

Fig. 4. (A): PCR (Genus primers). Lane1; Mol wt maker; lane 2. P. falciparum; lane 3, P. vivax; lane 4, P. Knowlesi; lane 5 -to- 13, clinical malaria samples. (B) PCR (Genus primers). Lane1; $P$. cynomolgi; lane 2, P. vivax; lane 3, P. falciparum (3D7); lane 4, P. falciparum (Dd2); clinical malaria samples:- lane 5. KS4; lane 6, KS3; lane7, MP20. (C). Gene Clean samples for sequencing. Lanes1-5, 1000bps band clinical samples viz G10A, KS3A, H9CA, B1A and B5A; lane 6, B5B (400bps); lane7, MP20A (1000Bps); lane8, MP20B (400Bps); lane 9, P. knowlesi (1000bp); lane10, P. knowlesi (400bps); lane11, P.cynamolgi(1000bp); lane12, $P$. cynamolgi(400bps); lane 13, P. knowlesi- new (400bp); lane 14, P. knowlesi-new (1000bp)

\subsection{BLAST Analysis of Nucleotide Sequences}

The raw DNA sequences were initially edited at the 5' and 3' prime end. Respective consensus sequences BLAST against NCBI non-redundant nucleotide database showed species-specific results. Each of the species specific primers derived sequences produced high score respective to the species (Table 1). The representative sequences from this work are deposited at NCBI nucleotide data base (GenBank accession id: KM263613.1 -toKM263613.1). BLASTn results based on $P$. falciparum specific primers produced expectation value (e-value $3 e^{-151}$ ) for 3D7 dynein light chain type 2, while the nearest neighbor, berghi, had e-value $4 \mathrm{e}^{-23}$ and there was no closely related human sequence observed (Table 1 (A). BLASTn output obtained from nucleotide sequences derived from Plasmodium genus specific primers showed interchangeable results where the high score for $P$. knowlesi dynein light chain type 2 had e-value $9 \mathrm{e}^{-158}$ and $P$. cynomolgi e-value $4 \mathrm{e}^{-112}$ (Table 1 ; (B) \& (C)) while $P$. vivax had the highest near neighbor score, e-value $1 \mathrm{e}^{-98}$ and no significant score against human derived nucleotide sequences. The genus primers targeted $P$. knowlesi sub-class and the BLASTn results showed this specificity. Similarly, BLASTn results from field clinical samples $P C R$ fragment sequence query (6PB and 7PB) had high score hits (e-value1 $\mathrm{e}^{-124}-1 \mathrm{e}^{53}$ ) within $P$. vivax, $P$. knowlesi and $P$. cynomolgi reflecting this target subclass of Plasmodium species (Table 2). 
Table 1. Nucleotide BLAST query results for respective Plasmodium species. The low Evalues observed in target species implies that the hits are significant. BLASTn default algorithm parameters (expectation thresh hold of 10)

Sequences producing significant alignments:

\section{(A). Plasmodium falciparum}

\section{Description}

P. falciparum 3D7 chromosome 11, complete

P. falciparum 3D7 DLC type 2, complete cds

P.berghei strain ANKA DLC type 2, mRNA

P.inui San Antonio 1 hypothetical protein mRNA

P.knowlesi strain H DLC type 2 complete cds

P.knowlesi strain H chromosome 9, complete genome

P.cynomolgi strain B DLC type 2 complete cds

P.vivax SaI-1 DLC type 2 partial mRNA

D. globosa $5.8 \mathrm{~S}$ rRNA gene $28 \mathrm{~S}$ rRNA

H. sapiens ankyrin repeat, chromosome 12

H. sapiens $12 \mathrm{BAC}$ RP11-179A1 complete sequence
E value

$3 \mathrm{e}^{-151}$

$3 \mathrm{e}^{-151}$

$4 \mathrm{e}^{-23}$

$8 \mathrm{e}^{-19}$

$4 \mathrm{e}^{-16}$

$4 \mathrm{e}^{-16}$

$2 \mathrm{e}^{-13}$

$2 \mathrm{e}^{-13}$

0.001

0.001

0.001
Accession

AE014186.2

XM_001347783.1

XM_673772.1

XM_008819688.1

XM_002259103.1

AM910991.1

XM_004222316.1

XM_001615210.1

KJ136787.1

NG_029860.1

AC079954.18

\section{(B). Plasmodium knowlesi}

\section{Description}

P.knowlesi strain H DLC type 2 complete cds

P.knowlesi strain H chromosome 9, complete

P.cynomolgi strain B DLC type 2 complete cds

P.vivax SaI-1 DLC type 2 partial mRNA

P.vinckeivinckei hypothetical protein mRNA

P.chabaudichabaudiDLC type 2, partial mRNA

P.yoeliiyoelii str. 17XNL partial mRNA

P.berghei strain ANKA DLC type 2 mRNA

S. lycopersicum chromosome, complete genome

G. max ubiquitin-protein ligase LIN-like mRNA

\section{(C). Plasmodium cynomolgi}

\section{Description}

P.knowlesi strain H DLC 2 complete cds

P.knowlesi strain H chromosome 9, complete

P.cynomolgistrain B DLC type 2complete cds

P.vivax SaI-1 DLC type 2 partial mRNA

P.inui San Antonio 1 hypothetical protein mRNA

T. versicolor $\mathrm{FP}-101664$ hypothetical protein mRNA

G. $\max$ E3 ubiquitin-protein ligase LIN-like mRNA

E value
$9 \mathrm{e}^{-158}$
$9 \mathrm{e}^{-158}$
$4 \mathrm{e}^{-112}$
$1 \mathrm{e}^{-98}$
0.016
0.016
0.057
0.057
0.69
0.69

Accession

XM_002259103.1

AM910991.1

XM_004222316.1

XM_001615210.1

XM_008626467.1

XM_732090.1

XM_721401.1

XM_673772.1

HG975521.1

XM_006586756.1

$\begin{array}{ll}\begin{array}{c}\text { E value } \\ 3 \mathrm{e}^{-146}\end{array} & \text { Accession } \\ 3 \mathrm{e}^{-146} & \text { XM_002259103.1 } \\ 5 \mathrm{e}^{-99} & \text { AM910991.1 } \\ 1 \mathrm{e}^{-86} & \text { XM_004222316.1 } \\ 1 \mathrm{e}^{-67} & \text { XM_001615210.1 } \\ 0.82 & \text { XM_008819688.1 } \\ 0.82 & \text { XM_008043674.1 } \\ & \text { XM_006586756.1 }\end{array}$


Table 2. Nucleotide BLAST query results for representative Malaria positive clinical samples

Sequences producing significant alignments:

(A). 6PB_TePfwd mp20B

\begin{tabular}{lll}
\multicolumn{1}{c}{ Description } & E value & \multicolumn{1}{c}{ Accession } \\
P. knowlesi DLC type 2 mRNA, complete cds & $3 \mathrm{e}^{-77}$ & XM_002259103.1 \\
P. knowlesi chromosome 9, complete genome & $3 \mathrm{e}^{-77}$ & AM910991.1 \\
$P$. vivax SaI-1 DLC type 2 partial mRNA & $1 \mathrm{e}^{-55}$ & XM_001615210.1 \\
$P$. cynomolgi DLC type 2 mRNA, complete cds & $7 \mathrm{e}^{-53}$ & XM_004222316.1 \\
$P$. inui hypothetical protein partial mRNA & $4 \mathrm{e}^{-43}$ & XM_008819688.1 \\
Feline coronavirus UU16, complete genome & $2 \mathrm{e}^{-15}$ & FJ938058.1 \\
Feline coronavirus RM, complete genome & $7 \mathrm{e}^{-09}$ & FJ938051.1
\end{tabular}

(B). 7PB_TcPfwd MB5b

\section{Description}

P. knowlesi DLC type $2 \mathrm{mRNA}$, complete cds

P. knowlesi strain $\mathrm{H}$ chromosome 9, complete genome

P. cynomolgi DLC type $2 \mathrm{mRNA}$, complete cds

$P$. vivax SaI-1 DLC type 2 partial $\mathrm{mRNA}$

$P$. inui hypothetical protein partial $\mathrm{mRNA}$

P. falciparum 3D7 chromosome 11, complete sequence

P. falciparum 3D7 DLC 2, putative mRNA, complete cds

$P$. vinckei vinckei hypothetical protein partial mRNA

P. chabaudi chabaudi DLC type 2, partial mRNA

$P$. yoelii genome assembly chromosome 9
E value

$1 \mathrm{e}^{-124}$

$1 \mathrm{e}^{-124}$

$4 \mathrm{e}^{-80}$

$4 \mathrm{e}^{-74}$

$4 \mathrm{e}^{-61}$

$5 \mathrm{e}^{-09}$

$5 \mathrm{e}^{-09}$

$3 \mathrm{e}^{-06}$

$3 \mathrm{e}^{-06}$

$1 \mathrm{e}^{-05}$
Accession

XM_002259103.1

AM910991.1

XM_004222316.1

XM_001615210.1

XM_008819688.1

AE014186.2

XM_001347783.1

XM_008626467.1

XM_732090.1

LM993663.1

\section{DISCUSSION}

This study presents new target gene for the detection of Plasmodium spp in human peripheral blood by parasite DNA amplification in polymerase chain reaction (PCR). In effort to address the need develop alternative target genes in detection of multiple species malaria infections, different laboratories have applied various PCR methods on 18S rRNA, cytochrome boxidase, circumsporozoite protein, tubulin and other genes [9,30-32]. Our previous in-silico studies [26] had shown that Plasmodium dlc-Tctex gene is a good target in diagnostic or drug development because the gene is conserved within the genus. The results presented in this study show that PCR targeting Plasmodium dynein light chain Tctex sub-unit can be applied to differentiate $P$. falciparum in cases of multiple malaria infections while application of the Plasmodium genus specific primers can discriminate between the species tested in this study: $P$. vivax, $P$. knowlesi and $P$. cynomolgi. When tested on microscope confirmed malaria blood samples, $P$. falciparum specific primers detected 11 positives out of the 30 samples and the target PCR band intensity was presumably, reflective of parasitaemia (Fig. 1a). The variation between microscopy observations and PCR results indicates other variant Plasmodium species are considered presumptive $P$. falciparum cases in routine clinical procedures.

Microscopic observation does not reliably distinguish between $P$. falciparum, $P$. vivax, $P$. knowlesi and $P$. cynomolgi where all these 
species are in transmission and is particularly important for zoonotic cases [33,34]. PCR done on in-vitro $P$. falciparum cultures (3D7/Dd2) and $P$. vivax, $P$. knowlesi and $P$. cynomolgi in-vivo controls showed that the species can be differentiated by PCR target gene band sizes, approximately $300 \mathrm{bps}$ and $400 \mathrm{bps}$ (Fig. 2a). When genus specific primers were applied on clinical malaria samples, both $P$. falciparum and non $P$. falciparum malaria targets and other undetermined products (Fig. 2(A), (B), (C)) were amplified. The genus primers could further be optimized to produce specific target as seen in Fig 2(C). Detection of Plasmodium infection in clinical malaria samples using genus primers amplified the approximately 300bps target PCR product (Fig. 3 (A), (B), (C)) and also a product approximately $1000 \mathrm{bps}$ coinciding with P. vivax controls (Fig. 3 (A), lane 3; Fig. $3(\mathrm{~B})$, lane 4; Fig. $3(C)$, lane 3). Some samples while positive for the $300 \mathrm{bps}$ PCR product were negative or showed a faint $1000 \mathrm{bps}$ product, indicating that the latter product reflect common $P$. vivax co- infection.

To determine the identity of the amplified PCR products malaria positive and control samples (Fig. $3(A),(B),(C)$ and $4(A),(B))$, some of the PCR products (Approx. 300bps- to $400 \mathrm{bps}$, and 1000bps see Mol wt maker Fig. $4(\mathrm{~A})$ and arrows Fig. 4(B), (C)) were gene cleaned for sequencing purposes, later discused. These target bands could be indicative of $P$. falciparum and non- $P$. falciparum malaria, while the 1000 bps product presumably corresponded to $P$. vivax infection. PCR data indicated presence of $P . f a l c i p a r u m$ and mixed infections in Kenya's malaria endemic areas and has implications on policy choice drug regimes in treatment of malaria. Presumptive $P$. falciparum cases are routinely treated with Artemensin- Lumefatrine or other combined therapy (ACT) while other species may be treated with Primaquine or Chloroquine [22,35]. Diagnosis of $P$. vivax and $P$. cynomolgi is required to administer antihyponozoite treatment to prevent relapses, while in $P$. knowlesi cases, urgency is required due to this species short replication period (12 hrs) that may lead to fatalities $[36,37]$. Some malaria endemic regions, for example; Papua New Guinea and Indonesia, have already adopted a unified treatment by ACT for all malaria cases $[19,38]$.

Prompt accurate diagnosis is precedence in the choice treatment of the different malaria cases. Currently available methods for the diagnosis of malaria in peripheral blood are parasite detection by microscopy, DNA or RNA detection methods by polymerase chain reaction (PCR), and detection of parasite antigens by rapid diagnostic tests (RDTs). The latter test is preferred because of its ease of use, relatively low cost and also detects circulating antigens even when the parasites are sequestered in the deep circulation [39-41]. There are concerns about RDTs test stability, accuracy, species detection, due to antigen genetic diversity, justifying the need to improve current RDT and further development of promising prototypes [12]. Moreover, new RTDs need to be pre-tested at PCR stage. Sequence results of the geneclean product of dlc-Tctex PCR target used in this study showed that the gene is unique to Plasmodium spp [26]. BLAST results of sequence query indicate that $P$. falciparum gene sequence is markedly different from other Plasmodium species: e-value1 $\mathrm{e}^{-151}$ compared to nearest neighbor e-value $1 \mathrm{e}^{-53}$ in $P$. berghi (Table 1, (A)) and had no significant similarity to any human gene sequence. This observation support the PCR results, where $P$. falciparaum primers had non-ambiguous single band target amplifications (Fig. 1a, Fig. 1b). BLAST e-values for sequence query from PCR primers targeting non- $P$. falciparum malaria indicate that Plasmodium species cluster differences; where, $P$. knowlesi and P. cynomolgi are in the same sub-group (Tables $1,(B) \&(C)$ ) and P.vivax dlc- Tctex sequence is very closely related. The expectation values scores were interchangeably similar (e-value $1 \mathrm{e}^{-158}$ and $1 \mathrm{e}^{-146}$ ) and distinct to the subgroup. This demonstrates the difficult in designing genus specific PCR primers for this sub-group. Malaria clinical samples amplified using primers targeting non- $P$. falciparum infection had similar sequence high score results within the sub-group, where $P$. knowlesi, $P$. cynomolgi and $P$. vivax cluster had relatively similar evalues (Tables 2, (A) \& (B)) indicating these primers can be applied to detect this subclass of Plasmodium species based on different molecular size products (Fig. 3 (A), (B), (C)). While the different species showed variation in molecular weight of the PCR band, the clinical malaria samples did not have high yield distinct bands and the sequencing results were not of particularly high quality. It is necessary to do further re-sequencing of optimized PCR products of targeted non- $P$. falciparum in mixed malaria infections to achieve better results. 
Transmission of mixed species infections in endemic regions is related to transmitting mosquito vectors and the proximity of the alternate host. In tropical and subtropical forested Southeast Asia, the importance of zoonoses malaria transmission buy non-human primates is increasingly being reported $[8,10,13]$. Such occurrence is expected in areas in subSaharan Africa, especially due to the close proximities between human and wild animals as people are encroaching forested areas for farming and urbanization. $P$. knowlesi has been considered simian malaria until recently when PCR diagnosis $[10,13,23]$ showed it is misdiagnosed for $P$. falciparum at ring stage and as $P$. malariae in other stages. Similarly, $P$. cynomolgi is indistinguishable from $P$. vivax and discriminative PCR is necessary to separate the two. The reality of mixed malaria infections requires effective diagnostic tools so that the right treatment strategies are followed and the government can then recommend a more rational drug treatment policy [21-22]. $P$. knowlesi has the shortest life cycle known for Plasmodium and this can lead to high parasitaemia and possible fatality [14] while $P$. cynomolgi presents hyponozoites which can initiate relapses just as the case in $P$. vivax [8]. The Target gene (dlc-Tctex) sequence BLAST results from clinical samples in this study indicate that $P$. falciparum and $P$. vivax mixed infection are likely, very common while $P$. knowlesi/P. cynomolgi zoonotic infections are probable in Kenyan human population in malaria endemic areas. Further refinement of this data will give clear evidence of the zoonoses transmission of primate malaria in this region.

\section{CONCLUSIONS}

PCR targeting Plasmodium dlc-Tctex gene can specifically detect $P$. falciparum and differentiate the species in mixed infection.

Various Plasmodium species have distinctive molecular size PCR band.

dlc-Tctex sequences BLASTn e-Value scores show distinctive clusters within Plasmodium spp and are markedly different compared to human orthologues.

\section{ACKNOWLEDGEMENTS}

Professor Raymond T. Damian (c/o Department of Cellular Biology, University of Georgia), reviewed and edited the manuscript.

\section{COMPETING INTERESTS}

Authors have declared that no competing interests exist.

\section{REFERENCES}

1. Luxemburger C, Nosten F, White N. Naturally acquired immunity to vivax malaria. Lancet. 1999;10:162.

2. Sachs J, Malaney $P$. The economic and social burden of malaria. Nature. 2002; 7(415):680-5.

3. Nosten $F$, Van Vugt $M$, Price $R$, Luxemburger C, Thway K, et al. Effects of artesunate- mefloquine combination onincidence of Plasmodium falciparum malaria and mefloquine resistancein western Thailand: A prospective study. Lancet. 2000;356:297-302.

4. Hartman T, Rogerson S, Fischer P. The impact of maternal malaria on newborns. Ann Trop Paediatr. 2010;30:271-82.

5. Cunha C, Cunha B. Brief history of the clinical diagnosis of malaria: From Hippocrates to Osler. J Vector Borne Dis. 2008;45:194-9.

6. Kappe S, Vaughan A, Boddey J, Cowman A. That was then but this is now: Malaria research in the time of an eradication agenda. Science. 2010; 14(328):862-6.

7. Lee G, Jeon E, Le D, Kim T, Yoo J, et al. Development and evaluation of a rapid diagnostic test for Plasmodium falciparum, $P$. vivax, and mixed-species malaria antigens. Am J Trop Med Hyg. 2011;85:989-93.

8. Ta T, Hisam S, Lanza M, Jiram A, Ismail $\mathrm{N}$, et al. First case of a naturally acquired human infection with Plasmodium cynomolgi. Malar J. 2014;24(13):68.

9. Collins W, Sullivan J, Jeffery G, Nace D, Williams $T$, et al. Mosquito infection studies with Aotus monkeys and humans infected with the Chesson strain of Plasmodiun vivax. Am J Trop Med Hyg. 2012;86:398-402.

10. Khim N, Siv S, Kim S, Mueller T, Fleischmann E, et al. Plasmodium knowlesi infection in humans, Cambodia, 2007-2010. Emerg Infect Dis. 2011;17: 1900-2.

11. Singh N, Kataria $O$, Singh $M$. The changing dynamics of Plasmodium vivax and $P$. falciparum in central India: Trends over a 27-year period (1975-2002). Vector Borne Zoonotic Dis. 2004;4:239-48. 
12. Chin W, Contacos $P$, Collins $W$, Jeter $M$, Alpert E. Experimental mosquito-transmission of Plasmodium knowlesi to man and monkey. Am J Trop Med Hyg. 1968;17:355-8.

13. Cox-Singh J, Singh B. Knowlesi malaria: Newly emergent and of public health importance? Trends Parasitol. 2008;24: 406-10.

14. Cox-Singh J, Hiu J, Lucas S, Divis $P$, Zulkarnaen M. Severe malaria - A case of fatal Plasmodium knowlesi infection with post-mortem findings: A case report. Malar J. 2010;11:9-10.

15. Marks M, Gupta-Wright A, Doherty F, Singer M, Walker D. Managing malaria in the intensive care unit. $\mathrm{Br} \mathrm{J}$ Anaesth. 2014; 113:910-921.

16. Mishra S, Panigrahi P, Mishra R, Mohanty S. Prediction of outcome in adults with severe falciparum malaria: A new scoring system. Malar J. 2007;6:24.

17. English M. Life-threatening severe malarial anaemia. Trans R Soc Trop Med Hyg. 2000;94:585-8.

18. Bruneel F, Tubach F, Corne P, Megarbane $B$, Mira J, et al. Severe imported malaria in adults (SIMA) study group. Severe imported falciparum malaria: A cohort study in 400 critically ill adults. PLoS One. 2010;5:e13236.

19. Marsh K, Forster D, Waruiru C, Mwangi I, Winstanley $M$, et al. Indicators of lifethreatening malaria in African children. $\mathrm{N}$ Engl J Med. 1995;332:1399-404.

20. World Health Organization, Guidelines for the Treatment of Malaria. Geneva: World Health Organization. 2010;1-194.

21. Ruizendaal E, Dierickx S, Grietens K, Schallig $H$, Pagnoni $F$, et al. Success or failure of critical steps in community case management of malaria with rapid diagnostic tests: A systematic review. Malar J. 2014;13:229

22. Dinko B, Oguike M, Larbi J, Bousema TC. Sutherland, persistent detection of Plasmodium falciparum, $P$. malariae, $P$. ovale curtisi and $P$. ovale wallikeri after ACT treatment of asymptomatic Ghanaian school-children. Int J Parasitol Drugs Drug Resist. 2013;3:45-50.

23. Chew $C$, Lim $Y$, Lee $P$, Mahmud R, Chua K. Hexaplex PCR detection system for identification of five human Plasmodium species with an internal control. J Clin Microbiol. 2012;50:4012-9.
24. Lucchi N, Demas A, Narayanan J, Sumari D, Kabanywanyi A, Kachur S, Barnwell J, Udhayakumar V. Real-time fluorescence loop mediated isothermal amplification for the diagnosis of malaria. PLoS One. 2010; 29(5):e13733.

25. Mixson-Hayden T, Lucchi N, Udhayakumar V. Evaluation of three PCR-based diagnostic assays for detecting mixed Plasmodium infection. BMC Res Notes. 2010;31(3):88.

26. Githui E, De Villiers E, McArthur A. Plasmodium possesses dynein light chain classes that are unique and conserved across species. Infect Genet Evol. 2009;9: 337-43.

27. Tai A, Chuang Z, Bode C, Wolfrum U, Sung C. Rhodopsin's carboxyterminal cytoplasmic tail act as a membrane receptor for cytoplasmic dynein by binding to the dynein light chain Tctex-1. Cell. 1999;97:877-87.

28. Altschul SF, Madden TL, Schäffer AA, Zhang J, Zhang Z, et al. Gapped BLAST and PSI-BLAST: A new generation of protein database search programs. Nucleic Acids Res. 1997;25(17):3389-402.

29. Schäffer L, Aravind T, Madden S, Shavirin $\mathrm{J}$, Spouge et al. Improving the accuracy of PSI-BLAST protein database searches with composition-based statistics and other refinements. Nucleic Acids Res. 2001;29:2994-3005.

30. malERA Consultative Group on Diagnoses and Diagnostics, A research agenda for malaria eradication: Diagnoses and diagnostics. PLoS Med. 2011;8:e1000396.

31. Barber B, William T, Grigg M, Piera K, Yeo $\mathrm{T}$, Anstey N. Evaluation of the sensitivity of a pLDH-based and an aldolase-based rapid diagnostic test for diagnosis of uncomplicated and severe malaria caused by PCR-confirmed Plasmodium knowlesi, Plasmodium falciparum and Plasmodium vivax. J Clin. Microbiol. 2013;51:1118-23.

32. Lucchi N, Poorak M, Oberstaller J, DeBarry J, Srinivasamoorthy G, et al. A new single- step PCR assay for the detection of the zoonotic malaria parasite Plasmodium knowlesi. PLoS One. 2012; 7(2):e31848.

33. Cox-Singh J, Davis T, Lee K, Shamsul S, Matusop A, et al. Plasmodium knowlesi malaria in humans is widely distributed and potentially life threatening. Clin Infect Dis. 2008;46:165-71. 
34. Barber B, William T, Grigg M, Yeo T, Anstey N. Limitations of microscopy to differentiate Plasmodium species in a region co-endemic for Plasmodium falciparum, Plasmodium vivax and Plasmodium knowlesi. Malar J. 2013; 8(12):8.

DOI: $10.1186 / 1475-2875-12-8$

35. Barber B, William T, Grigg M, Menon J, Auburn S, et al. A prospective comparative study of knowlesi, falciparum, and vivax malaria in Sabah, Malaysia: High proportion with severe disease from Plasmodium knowlesi and Plasmodium vivax but no mortality with early referral and artesunate therapy. Clin Infect Dis. 2013;56:383-97.

36. William T, Menon J, Rajahram G, Chan L, Ma G, et al. Severe Plasmodium knowlesi malaria in a tertiary care hospital, Sabah, Malaysia. Emerg Infect Dis. 2011;17:124855.

37. William $\mathrm{T}$, Rahman $\mathrm{H}$, Jelip J, Ibrahim $\mathrm{M}$, Menon $\mathrm{J}$, et al. Increasing incidence of Plasmodium knowlesi malaria following control of $P$. falciparum and $P$. vivax Malaria in Sabah, Malaysia. PLoS Negl Trop Dis. 2013;7:e2026.

38. Mombo-Ngoma G, Kleine C, Basra A, Würbel $H$, Diop $D$, et al. Prospective evaluation of artemether-lumefantrine for the treatment of non-falciparum and mixed-species malaria in Gabon. Malar J. 2012;11:120.

DOI: 10.1186/1475-2875-11-120

39. Mason $\mathrm{D}$, Kawamoto $\mathrm{F}$, Lin $\mathrm{K}$, Laoboonchai A, Wongsrichanalai C. A comparison of two rapid field immunochromatographic tests to expert microscopy in the diagnosis of malaria. Acta Trop. 2002;82:51-9.

40. Davis T, Mueller I, Rogerson S. Prevention and treatment of malaria in pregnancy. Future Microbiol. 2010;5:1599-613.

41. Gillet $\mathrm{P}$, Mukadi $\mathrm{P}$, Vernelen $\mathrm{K}$, Van Esbroeck M, Muyembe J, et al. External quality assessment on the use of malaria rapid diagnostic tests in a non-endemic setting. Malar J. 2010;9:359. DOI: 10.1186/1475-2875-9-359

(c) 2019 Kariuki et al.; This is an Open Access article distributed under the terms of the Creative Commons Attribution License (http://creativecommons.org/licenses/by/4.0), which permits unrestricted use, distribution, and reproduction in any medium, provided the original work is properly cited.

Peer-review history:

The peer review history for this paper can be accessed here: http://www.sdiarticle3.com/review-history/50344 